\title{
Monitoreo de la evapotranspiración real horaria por medio del balance energético y un lisímetro de pesada
}

\author{
Adán Matías Gabriel Faramiñán ${ }^{(1,2)}$, Facundo Carmona ${ }^{(1,2)}$, Raúl Eduardo Rivas ${ }^{(1,3)}$, María Florencia \\ Degano $^{(1,3)}$ y Paula Olivera Rodríguez ${ }^{(1,2)}$
}

(1) Instituto de Hidrología de Llanuras. CIC-CONICET-UNCPBA. Gral. Pinto 399, C.P. B7000GHG, Tandil, Buenos Aires, Argentina. adanfaraminian@ihlla.org.ar

(2) Consejo Nacional de Investigaciones Científicas y Técnicas (CONICET)

(3) Comisión de Investigaciones Científicas de la Provincia de Buenos Aires (CICPBA)

\begin{abstract}
RESUMEN
Conociendo adecuadamente en espacio y tiempo la evapotranspiración es posible gestionar y planificar el manejo hídrico que se le da a una cuenca de llanura. Se estudia de forma indirecta a escala local por medio de estaciones agrometeorológicas, o a escala regional con el uso de datos de satélite. Para tener fiabilidad de los modelos utilizados, es de suma importancia validar la información generada con medidas directas. De acuerdo con esto, se presenta el estudio de medidas directas de evapotranspiración en suelos arcillosos cubiertos por gramíneas en condiciones de secano para la validación de un modelo simple de balance energético en la región Pampa húmeda del centro-oeste de la Argentina. Para ello, se utilizaron registros horarios de una estación de balance de energía y se relacionaron con medidas directas de un lisímetro de pesada durante los meses de febrero-marzo y septiembre de 2017. Con el método energético se obtuvieron valores aproximados de evapotranspiración real para registros máximos de humedad edáfica $\left(0,01-0,15 \mathrm{~mm} \mathrm{hora}^{-1}\right)$, mostrando mayores diferencias $\left(0,1-0,35 \mathrm{~mm}_{\text {hora }}{ }^{-1}\right)$ cuando la humedad del suelo tiende a valores mínimos. En este trabajo se demostró que un modelo simplificado del balance de energía para determinar la evapotranspiración como flujo residual es aplicable para áreas con coberturas de referencia definidas (Allen et al., 1998), permitiendo simplificaciones en las medidas de resistencias aerodinámicas y del cultivo. Este aporte brinda información necesaria para la validación de modelos satelitales aplicados a zonas de llanura, en donde la evapotranspiración es un término de relevancia.
\end{abstract}

Palabras clave: Balance de energía, Humedad del suelo, Lisímetro, Llanura.

\section{Monitoring the hourly actual evapotranspiration through the energy balance and a weighing lysimeter}

\begin{abstract}
By having sufficient knowledge about evapotranspiration in space and time, it is possible to manage and plan the water management of a basin. Evapotranspiration can be obtained indirectly at a local scale through information recorded in agrometeorological stations, or regionally with the use of satellite data. In order to have reliable models, it is very important to validate the information generated with direct measures of evapotranspiration. Therefore, direct measurements of evapotranspiration in clay secano-soils covered by grasses and the use of the data generated for the validation of indirect measurements are presented in this study. To achieve this, hourly data from an energy balance station and direct measurements of a weighing lysimeter were used to study the water loss during two experimental campaigns carried out in Tandil (Argentina), in the months February-March and September 2017. The comparison of the methods was made for different moisture conditions of the soil. The energy balance model estimates approximate values of actual evapotranspiration for maximum values of soil moisture (0.01-0.15 mm hour-1), showing greater differences (0.1-0.35 $\mathrm{mm}$ hour-1) when the soil moisture tends to minimum values. However, it was demonstrated that a simple
\end{abstract}


Faramiñán, A.M.G., et al., 2021. Monitoreo de la evapotranspiración real horaria por medio.... Boletín Geológico y Minero, 132 (1-2): 47-56

one-level model to determine the latter variable is applicable for areas with reference coverage, allowing simplifications in aerodynamic and crop resistance measurements (Allen et al. 1998). This contribution provides necessary information for the validation of satellite models applied to plains areas, where evapotranspiration is a term of great importance.

Keywords: energy balance, lysimeter, plains area, soil moisture.

\section{Introducción}

La evapotranspiración ( $E T)$ se define como la combinación de dos procesos separados por los que el agua se pierde a través de la superficie del suelo por evaporación y, por otra parte, mediante transpiración desde la vegetación. Este término hidrológico tiene una gran importancia, por ejemplo, en el sistema de llanura de la Pampa húmeda-subhúmeda de la Argentina aproximadamente $80 \%$ del agua que precipita se pierde como evapotranspiración (Weinzettel y Usunoff, 2001). La ET puede determinarse midiendo varios componentes del balance de agua en el suelo. El método consiste en evaluar los flujos de agua que entran y salen de la zona radicular del cultivo dentro de un determinado periodo de tiempo (Allen et al. 1998). La $E T$ se define de la siguiente manera:

$$
E T=R+P-E S-D+C \pm \Delta F S \pm \Delta S
$$

donde el riego $(R)$ y la precipitación $(P)$ proporcionan agua a la zona radicular. Parte de $\mathrm{R}$ y $\mathrm{P}$ pueden perderse por escurrimiento superficial (ES), mientras que el drenaje profundo (D) eventualmente recargará el acuífero. El agua también puede moverse hacia la superficie mediante capilaridad $(C)$ desde la capa freática sub-superficial hacia dentro o fuera de la zona radicular $(\Delta \mathrm{FS})$. Sin embargo, excepto bajo condiciones de pendientes pronunciadas, normalmente $\Delta \mathrm{FS}$ puede despreciarse. La evaporación del suelo y la transpiración de la vegetación pueden agotar el agua de la zona radicular. Algunos flujos, como el flujo sub-superficial, el drenaje profundo y la capilaridad desde la capa freática, son difíciles de medir y pueden no considerarse en periodos cortos de tiempo. Si se aísla la zona radicular de la vegetación y se controlan los procesos que son difíciles de medir, los diversos términos de la ecuación del balance de agua se pueden determinar con precisión. Esto se puede realizar con lisímetros de pesada (LP), los cuales miden la pérdida de agua directamente por el cambio de masa $(\Delta S)$ en cortos intervalos de tiempo, desde horas a minutos (Aboukhaled et al., 1986; Parisi et al., 2009). Por lo tanto, los lisímetros permiten obtener medidas reales de ET (Pereira et al., 2004; Allen et al., 2011a y 2011b), P (Schrader et al., 2013; Hoffmann et al., 2015), D y niveles freáticos (Olías et al., 2003; Schwaerzel and Heiko, 2003; Fahle and Dietrich, 2014; Ma et al., 2019; Dietrich et al., 2019).

La $E T$ es controlada por el intercambio de energía en la superficie de la vegetación y es limitada por la can- tidad de energía disponible (Chow, 1994; Brutsaert, 2005). Debido a esta limitación, la $E T$ se puede medir aplicando el principio de conservación de la energía. Todos los flujos de energía deben ser considerados cuando se deriva una ecuación de balance de energía $(B E)$, y se relacionan de la siguiente manera:

$R n=H+G+L E+\Delta S+D_{h}+P h$

donde $R n$ es la radiación neta, $H$ es el flujo de calor sensible, $G$ es el flujo de calor del suelo, $L E$ es el flujo de calor latente, $\Delta S$ es el almacenamiento de calor y $P h$ es la energía que requiere la vegetación para realizar la fotosíntesis. Los términos el calor almacenado y liberado de la planta, la energía utilizada en actividades metabólicas o energía que se transfiere horizontalmente por advección solo utilizan una pequeña fracción de la radiación neta diaria y se pueden despreciar cuando se comparan con los otros cuatro componentes. Los distintos términos de la Ecuación 2 pueden ser positivos o negativos, dependiendo de las condiciones de la superficie (Carmona et al. 2011). Las Ecuaciones 1 y 2 se relacionan por los términos $E T$ y $L E$. $L E$ representa la energía que se invierte en el proceso de $E T$, la cual puede derivarse de la ecuación de $B E$ si todos los otros componentes son conocidos. Los valores de $R n$ y $G$ se pueden medir de manera directa por medio de un radiómetro neto y una placa de flujo de calor respectivamente. Por el contrario, la obtención de medidas de $H$ es compleja debido a que incluye mediciones precisas de los gradientes de temperatura y viento por encima de la superficie (Brutsaert, 2005; Ibañez Plana, 1998).

Es complejo monitorear el balance hidrológico con métodos de medida puntual en un área extensa con diferentes coberturas vegetales, lo que implica diferentes tasas de pérdida de agua en la zona no saturada. En la actualidad para obtener una estimación de la distribución espacial de los flujos de agua saliente y entrantes en la zona saturada, se utilizan modelos que parten del balance de energía utilizando datos procedentes de teledetección (Carmona et al. 2011; Holzman et al., 2014; Carmona et al. 2018). Por lo tanto, este trabajo se centró en la determinación de la $E T$ a través de un lisímetro de pesada con el objetivo de validar los datos obtenidos por un método de balance de energía simplificado. Se analizó principalmente la variación horaria del almacenamiento del agua en la zona no saturada motivada por la escasa información que existe sobre este enfoque. En la relación de los modelos se tuvo en cuenta condiciones de alta y baja humedad de suelo. El estudio se realizó en una zona 
con predominio de suelos Argiudoles de la región centro-oeste de la República Argentina. Los datos utilizados fueron registrados por una Estación de Lisímetro de Pesada con registro Continuo $(E L P C)$ y una de Balance de Energía ( $E B E$ ) en los meses de febrero, marzo y septiembre del 2017. Este aporte pretende brindar información necesaria para la validación de modelos satelitales aplicados a zonas de Ilanura, en donde la evapotranspiración es un término de gran importancia.

\section{Zona de estudio}

El estudio se realizó dentro de la parcela experimental ubicada en el Campus Universitario de la ciudad de Tandil $\left(37^{\circ} 17^{\prime}\right.$ de latitud Sur y $59^{\circ} 07^{\prime}$ de longitud Oeste, $214 \mathrm{~m}$ de altitud), de la Universidad Nacional del Centro de la provincia de Buenos Aires, que mantiene el Instituto de Hidrología de Llanura “Dr. Eduardo Usunoff" (IHLLA) desde el año 2005 (Figura 1). La zona posee un clima templado húmedo-subhúmedo, con precipitación promedio anual (1970-2010) de $925 \mathrm{~mm}$ y una evapotranspiración real de entre 730 y $820 \mathrm{~mm}$ año-1 $^{-1}$ (Ocampo et al. 2013; Aliaga et al. 2017). El nivel freático en el área de estudio se ubica a $5 \mathrm{~m}$ por debajo de la superficie y, por la profundidad a la que se encuentra, el proceso de evaporación y transpiración es independiente de éste (Fahle and Dietrich, 2014; Ma et al. 2019). El suelo corresponde a un Argiudol Típico (Silicani, 2014). La estructura y composición del suelo se explican en la Tabla 1. El suelo muestra características de un suelo Serie Tandil (Ta) (INTA, 1956-2019). Según la clasificación del Instituto Nacional de Tec- nología Agropecuaria (INTA) el suelo tiene aptitudes agrícolas y/o ganaderas.

En la parcela predominan tres especies en la cobertura vegetal (Dactylis glomerata, Festuca Arundinacea y Lolium multiflorum). La altura de la cubierta se mantuvo entre 0,10 y $0,14 \mathrm{~m}$ durante toda la campaña. En el área circundante se encuentran las especies mencionadas anteriormente y un área experimental de pastoreo de ganado vacuno (Schirmbeck and Rivas, 2007). En los periodos comprendidos en otoño y primavera, la zona experimental se caracteriza por tener la misma altura que la pastura de la parcela $(0,12 \mathrm{~m})$ permitiendo simplificaciones en el cálculo de la resistencia aerodinámica propuesto por Allen et al. (1998). Por este motivo se eligió estudiar la pérdida de agua en los meses de febrero, marzo y septiembre del 2017.

\section{Materiales}

El desarrollo de la ELPC consto de una parte edáfica y otra electrónica. La primera parte corresponde a la construcción de este lisímetro, basándose en la definición de Aboukhaled et al. (1986), Parisi et al. (2009) y en el trabajo de tesis de especialización en riego y drenaje de Silicani (2014), pero con la diferencia que no se perturbó el suelo en su instalación, conservando la estructura a monitorear, su biota, la pastura natural y por ende las raíces de la misma. Esta práctica tiene la ventaja de mantener las características biológicas, texturales y estructurales originales del suelo y no tener que esperar tiempos largos (8-10 meses, Silicani, 2014) a que se reestablezcan las condiciones naturales (Faramiñán et al., 2017). El cilindro del lisímetro

\begin{tabular}{ccccccc}
\hline Horizonte & Profundidad $(\mathrm{cm})$ & MO\% & \%Arcilla & \%Limo & \%Arena & Clase Textural \\
\hline Ap1 & $0-7$ & 5,69 & 26 & 37 & 37 & Franca \\
Ap2 & $7-20$ & 4,00 & 26 & 38 & 36 & Franca \\
AB & $20-35$ & 2,08 & 27 & 36 & 37 & Franca \\
Bt1 & $35-57$ & 1,45 & 45 & 26 & 29 & Arcillosa \\
Bt2 & $57-70$ & 0,79 & 40 & 25 & 35 & Arcillosa \\
BC & $70-78$ & 0,52 & 20 & 36 & 44 & Franca \\
C & $78-90$ & 0,34 & 8 & 39 & 53 & F. Arenosa \\
\hline
\end{tabular}

Tabla 1. Descripción de la morfología y composición del suelo característico de la zona de estudio.

Table 1. Description of the morphology and soil composition of the study area. 
posee un diámetro de 0,59 $\mathrm{m}, 0,003 \mathrm{~m}$ de espesor y $0,55 \mathrm{~m}$ de alto, de los cuales los $0,05 \mathrm{~m}$ de la base corresponden a la estructura de drenaje. Esta última está diseñada para facilitar la percolación profunda del excedente de agua. La profundidad seleccionada queda definida por la exploración de raíces y no supera los 0,5 m (Dormaar y Chanasyk, 1995). Debajo del lisímetro se encuentra una balanza (compuesta por una celda de carga) que soporta hasta $300 \mathrm{~kg}$ (con una precisión de $0,05 \mathrm{~kg}$ ), con el objetivo de registrar el
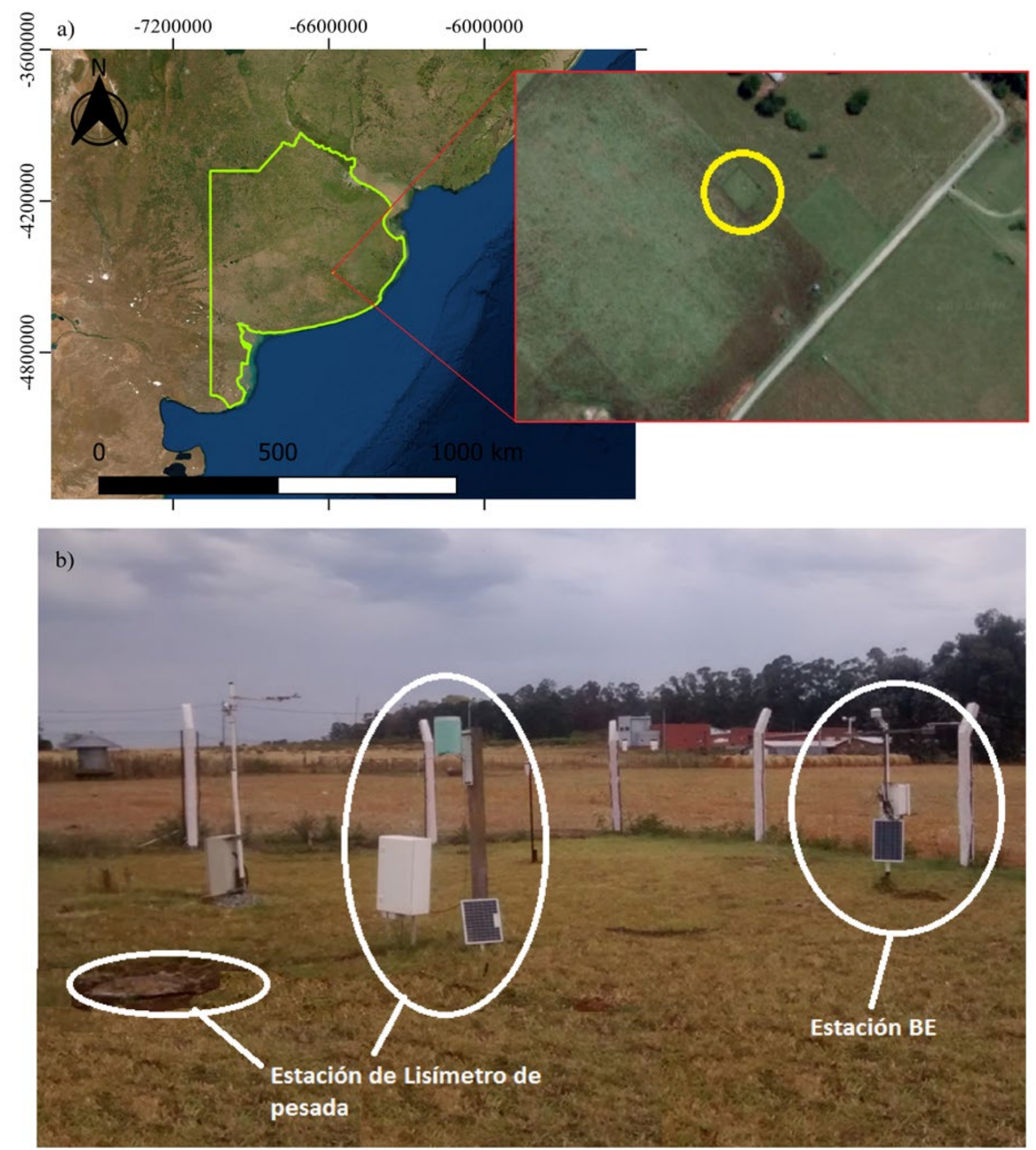

Figura 1. En (a) se muestra una imagen satelital de la zona de llanura estudiada y otra imagen aérea de la parcela de estudio (19x16 m). En (b) se muestra una imagen de la parcela experimental del IHLLA donde se indica la Estación del Lisímetro de Pesada y la Estación de Balance de Energía.

Figure 1. In (a) a satellite image of the studied plain area and another aerial image of the study zone are shown. In (b) an image of the experimental plot of the IHLLA is shown, where the weighing lysimeter and the energy balance stations are indicated. 
peso de suelo a estudiar. La comparación de la humedad del lisímetro con el entorno se realizó por medio de dos sensores de humedad pertenecientes a otra estación en la misma parcela. Los sensores cubrieron una profundidad de $0,40 \mathrm{~m}$ y registraron datos cada 1 hora. El desarrollo electrónico de la ELPC consistió en la configuración de la balanza. La misma se conectó a un datalogger $(D L)$ para almacenar las medidas del peso del lisímetro con una resolución temporal de 1 hora. Los datos se descargan directamente desde el $D L$ mediante una computadora.

Por otra parte, en relación con el balance de energía, la $E B E$ se compuso por diferentes sensores (Tabla 2 , y las características específicas de cada sensor se muestran en Carmona et al., 2011). Un datalogger se configuró para almacenar cada hora los valores medios, máximos, mínimos y desviaciones estándar de cada variable. Se utilizó la energía de una batería de $12 \mathrm{~V}$ alimentada con un panel solar de $20 \mathrm{~W}$ para su recarga. Al igual que en ELPC, los datos son descar- gados del $D L$ con una computadora. En la Tabla 2 se detallan los sensores que componen la ELPC y $E B E$ además de una breve descripción de la instalación.

\section{Métodos}

A partir del balance de masa o balance hídrico en un perfil del suelo se puede determinar la pérdida de agua y así obtener valores reales de $E T\left(E T_{\text {real }}\right)$. Aislando la zona radicular, la Ecuación 1 se simplifica de la siguiente manera

$$
E T_{L I}=P-D \pm \Delta S
$$

donde $E T_{L I}$ es la evapotranspiración medida por el lisímetro. Cuando no se registran precipitaciones y, por consecuencia, tampoco se registra drenaje profundo, la pérdida de agua por ET es directamente proporcional a $\Delta S$, definida de la siguiente manera

$$
\Delta S=C_{L I}\left(P e_{i}-P e_{i-1}\right)
$$

\begin{tabular}{|c|c|c|c|c|}
\hline & Instrumento & Modelo & Precisión & Instalación \\
\hline \multirow{5}{*}{ 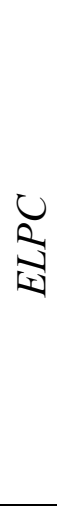 } & Balanza de piso & $\mathrm{SH}$ & $\pm 50 \mathrm{~g}$ & $\begin{array}{l}\text { Compuesta por una celda de carga, se } \\
\text { instaló debajo del tanque. }\end{array}$ \\
\hline & $\begin{array}{l}\text { Cilindro de hierro } \\
\text { dúctil }\end{array}$ & & & $\begin{array}{l}\text { Contenedor del suelo. Una mejor } \\
\text { descripción en Faramiñán et al. } 2017\end{array}$ \\
\hline & $\begin{array}{l}\text { Placa de flujo de } \\
\text { calor }\end{array}$ & HFT3 & $\pm 5 \%$ & $\begin{array}{c}\text { Se encuentra a } 0,1 \mathrm{~m} \text { de profundad dentro } \\
\text { del lisímetro. }\end{array}$ \\
\hline & $\begin{array}{l}\text { Sensores de } \\
\text { humedad }\end{array}$ & $\mathrm{SCH}-20$ & $\pm 3 \%$ & $\begin{array}{l}\text { Tres sensores que cubren } 0,40 \mathrm{~m} \text { de } \\
\text { profundidad. }\end{array}$ \\
\hline & Datalogger & CR800 & & \\
\hline \multirow{8}{*}{$\underset{10}{\frac{10}{10}}$} & Radiómetro neto & CNR2 & $<1 \%$ & Se ubicó a una altura de $2 \mathrm{~m}$. \\
\hline & $\begin{array}{l}\text { Temperatura } \\
\text { radiativa }\end{array}$ & SI-111 & $\pm 0,2{ }^{\circ} \mathrm{C}$ & $\begin{array}{c}\text { Se ubicó a una altura de } 2 \mathrm{~m} \text {, inclinado a } \\
45^{\circ} \text { en referencia a la vertical }\end{array}$ \\
\hline & $\begin{array}{l}\text { Temperatura del } \\
\text { aire }\end{array}$ & CS215 & $\pm 0,4{ }^{\circ} \mathrm{C}$ & Se ubicó a una altura de $2 \mathrm{~m}$. \\
\hline & Humedad relativa & CS215 & & Se ubicó a una altura de $2 \mathrm{~m}$. \\
\hline & $\begin{array}{l}\text { Velocidad del } \\
\text { viento }\end{array}$ & 014A & $1,5 \%$ & Se ubicó a una altura de $2 \mathrm{~m}$. \\
\hline & Pluviómetro & RG600 & $\pm 0,2 \mathrm{~mm}$ & Se ubicó a una altura de 1,5 m. \\
\hline & $\begin{array}{l}\text { Flujo de calor del } \\
\text { suelo }\end{array}$ & HFP01 & $\pm 2 \%$ & Se encuentra a $0,1 \mathrm{~m}$ de profundad. \\
\hline & Datalogger & CR1000 & & \\
\hline
\end{tabular}

Tabla 2. Detalle de los instrumentos utilizados, modelos, precisión e instalación. Table 2. Details of the instruments, models, measurement accuracy and set up. 
donde el termino $\Delta S$ se define por medio de lisímetros. $P e_{i}$ y $P e_{i-1}$ se traducen en pesadas por tiempo y el subíndice i equivale al tiempo que se registra la medición (en horas para este trabajo). El $C_{L I}$ es un factor de conversión que tiene en cuenta las dimensiones del lisímetro con el fin de conseguir valores en $\mathrm{mm}$ $\mathrm{h}^{-1}$. En este trabajo $C_{4}$ tiene un valor de $3,411 \mathrm{~mm} \mathrm{~kg}^{-1}$.

Por otro lado, analizando las energías impulsoras del balance hidrológico a partir de la Ecuación 2, se calculó $L E$ cómo término residual, despreciando los términos de almacenamiento, advección y fotosíntesis. Con respecto a los dos primeros tienen valores muy bajos en comparación con los demás. Los días con fuerte advección se descartaron según el criterio adoptado por Pereira et al., 2004, siguiendo la siguiente expresión: $0,5<E T_{L /} / R n_{+}<0,9$, en donde $R n_{+}$ representa los valores diurnos de la radiación neta. Tanto $R n$ como $G$, se obtuvieron de manera directa por medio de sensores de la $E B E\left(R n_{B E}\right.$ y $G_{B E}$ respectivamente). La diferencia entre estos términos determina la energía que se pierde en forma de calor latente y sensible. En consecuencia, la precisión del método se centra en estimar valores adecuados de $H$. En la actualidad no existe un sensor o dispositivo que determine de forma directa el término $H$, siendo el motivo del por qué se determinó a través de un modelo empírico. Se consideró $H$ proporcional a la diferencia de temperatura entre dos niveles en la subcapa inercial (teoría de la similitud de Monin-Obukhov), siendo proporcional a la diferencia entre la temperatura radiativa y la temperatura del aire a nivel de referencia, e inversamente proporcional a la resistencia aerodinámica. Estos modelos en los que una sola temperatura de la superficie dirige todo el proceso de intercambio se conocen como modelos de un nivel (Ibañez Plana, 1998). El método propuesto explica la relación suelo-vegetación-atmósfera de forma simple y sin la necesidad de una gran cantidad de instrumentos. La $E T$ (en unidades de $\mathrm{mm} \mathrm{h}^{-1}$ ) se obtiene de la siguiente manera:

$E T_{B E}=C_{B E}\left(R n_{B E}-G_{B E}-\left[\frac{\rho_{a} C_{p}}{r_{a h}}\left(T_{\text {aero }}-T_{a}\right)\right]\right)$

donde $E T_{B E}$ es la evapotranspiración obtenida como termino residual del balance de energía, $C_{B E}$ es un factor de conversión para tener medidas en $\mathrm{mm} \mathrm{h}^{-1}$ (Allen et al. 1998), $\rho_{a}$ es la densidad media del aire (CIPM-2007, Picard et al., 2008), $C_{p}$ es el calor específico del aire, $T_{\text {aero }}$ es la temperatura aerodinámica de la superficie, $\stackrel{a}{a}_{a}$ es la temperatura del aire y $r_{a h}$ es la resistencia aerodinámica de la vegetación. Los términos que están en corchetes representan el modelo de una capa. De acuerdo a Ibañez Plana (1998), en este trabajo se sustituye $T_{\text {aer }}$ por la temperatura radiativa $\left(T_{\text {rad }}\right)$, con el fin de tener menores errores en casos de cubiertas no completas. La $T_{\text {rad }}$ de la superficie se relaciona de forma directa con $T_{\text {rad }}$ de suelo y vegetación. En este trabajo el término $T_{\text {rad }}^{\text {rad }}$ se midió directa- mente con en el sensor SI-111 (Apogee Instruments).

El término $r_{a h}$ se determinó a partir del trabajo de Allen et al. (1998) presentado por la FAO. Para una altura de la cubierta de 0,12 m la resistencia aerodinámica se simplifica en los términos $208 / u_{2}$, donde $u_{2}$ es la velocidad del viento a $2 \mathrm{~m}$ de altura. Diferentes autores muestran que estos modelos no son precisos si no se introduce una corrección a la resistencia aerodinámica (Ibañez Plana, 1998). Para que esto se cumpla, y como se mencionó anteriormente, se estudiaron periodos en donde la altura de cobertura vegetal circundante y la pastura dentro de la parcela experimental fueran la misma.

Se utilizó la teoría de la propagación de errores para definir la incertidumbre de la $E T_{B E}$ por medio de la siguiente expresión,

$\Delta E T_{B E}=\Delta R n_{B E}+\Delta G_{B E}+\frac{\partial H}{\partial \rho_{a}} \Delta \rho_{a}+\frac{\partial H}{\partial T_{\text {rad }}} \Delta T_{\text {rad }}+\frac{\partial H}{\partial T_{a}} \Delta T_{a}+\frac{\partial H}{\partial u_{2}} \Delta u_{2}$

donde $\Delta$ indica la incertidumbre del término. Este error es propio de cada instrumento.

Para calcular la sensibilidad de la respuesta a los cambios en los parámetros, se realizó un análisis de la varianza (ANOVA) de las variables que intervienen en la Ecuación 6 con el fin de explicar la ETreal. Se utilizó un modelo lineal generalizado para explicar la igualdad entre las medias de la variable y se trabajó con un nivel de significancia de 0.05 . El peso de cada parámetro en la sensibilidad se mide por la correlación entre la variable y la respuesta. Las diferencias estadísticamente significativas en esta correlación indican variables sensibles a los cambios de $E T$.

\section{Resultados y discusión}

En la Figura 2 se muestran datos de ET obtenidos por medio las Ecuaciones 4 y 6 . Además, se muestran valores de humedad del suelo medidos por sensores para el mismo periodo. En estos últimos también se observan saltos que representan eventos de precipitación. En el periodo se registraron periodos secos con valores cercanos a $0,2 \mathrm{~m}^{3} \mathrm{~m}^{-3}$, periodos húmedos con registros de $0,41 \mathrm{~m}^{3} \mathrm{~m}^{-3}$ y una precipitación total de $161 \mathrm{~mm}$.

Para valores de $\mathrm{Hs}$ por debajo de $0,30 \mathrm{~m}^{3} \mathrm{~m}^{-3}$ se observó que los modelos muestran diferencias, principalmente en horas de máxima $R n$. En estos casos los datos de $E T_{B E}$ sobrestimaron a los de $E T_{L}$. Sin embargo, se observa que para valores de humedad mayores a $0,35 \mathrm{~m}^{3} \mathrm{~m}^{-3}$ los modelos se asemejan, registrándose diferencias menores a $0,15 \mathrm{~mm} \mathrm{~h}^{-1}$ para valores de humedad cercanos a $0,4 \mathrm{~m}^{3} \mathrm{~m}^{-3}$ (valores máximos de $\mathrm{Hs}$ ).

En la Figura 2, representados con una cruz, se muestran casos en donde advecciones fuertes afectan las medidas, resultando un ejemplo el día 50 . Las cruces también representan medidas nocturnas, en donde la $E T$ se considera despreciable. Lógicamente, con fuertes advecciones las diferencias entre los métodos crecen, sin embargo, se demuestra que el criterio puede 
utilizarse también para medidas horarias.

Por otro lado, a escala diaria y teniendo en cuenta que la ET nocturna es despreciable, en la Figura 3 se muestran las medidas diurnas para evaluar la ETBE y ETLI (0-6 mm día-1). En esta comparación se agregaron datos de septiembre del mismo año. En días con suelo húmedo (Hs>0,35 m3 m-3) los valores de ETLI y ETBE se asemejan y se posicionan cerca de la línea
1:1 y dentro del intervalo marcado por la desviación típica. Los valores que están por debajo del extremo inferior representan días en donde el perfil del suelo estaba con baja disponibilidad de agua $(\mathrm{Hs}<0,3 \mathrm{~m} 3$ $\mathrm{m}-3)$. En esta relación, siguiendo el criterio para despreciar mediciones afectadas por fuerte advección, quedaron 29 datos de los 47 que se disponían. En el análisis de incertidumbre se observó mínimas difer-
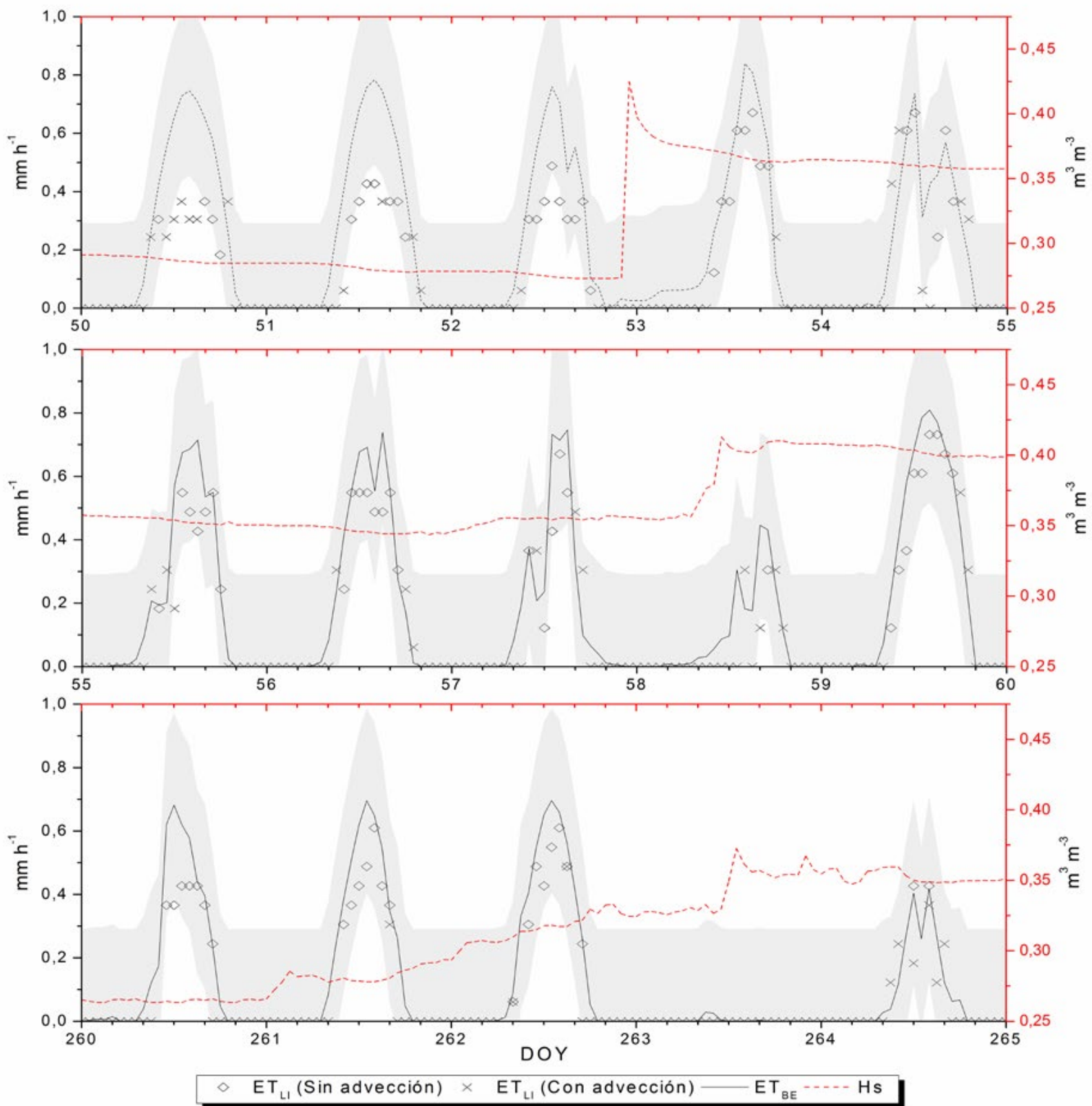

Figura 2. Registros de $H s$ y evapotranspiración ( $E T_{B E}$ y $E T_{L l}$ ) para el periodo comprendido entre los días del año 50-59 y 260-264 (DOY por su sigla en inglés). Los marcadores esféricos detallan medidas que no fueron afectadas por advección, los casos contrarios se muestran con una cruz. El área gris marca la incertidumbre de $E T_{B E}$.

Figure 2. Soil moisture (Hs) and evapotranspiration records ( $E T_{B E}$ and $\left.E T_{4}\right)$ for the period between DOY 50-59 and 60-64. The spherical markers detail measures that were not affected by advection, the opposite cases are shown with a cross. The grey area indicates the uncertainty of the $E T_{B E^{*}}$ 
encias para $E T_{B E}$ según la escala temporal. Para valores horarios, en casos de máxima radiación el valor residual de la $E T_{B E}$ tiene valores aproximados del $30 \%$. Valores similares se calcularon para los registros diarios, en donde el error fue del $33 \%$ de la medida.

Desde el punto de vista energético, la diferencia más notoria entre los datos diarios y horarios lo da el aporte del término $G$. Este parámetro se suele despreciar a escala de días o semanas, pero cuando se aumenta la resolución temporal se encontró que $G$ puede alcanzar hasta el $18 \%$ de $R n$. Por otra parte, anteriormente se describió la relación de los métodos con valores altos de $\mathrm{Hs}$. Para estos se puede afirmar que la energía disponible $(R n-G)$ se pierde mayoritariamente como $E T$, en otras palabras, la $E T_{B E}$ se asemeja a valores de $E T_{\text {real }}$ En los casos donde $H s$ tiende a valores mínimos, la energía disponible se utiliza en gran parte para el proceso de flujo de calor sensible, por lo que $H$ aumenta y en consecuencia la $E T$ disminuye. Sin embargo, se observan mayores diferencias en la relación de los valores de $E T_{B E}$ con registros del lisímetro en condiciones de baja $H s$.

Se realizó un análisis exhaustivo del modelo ener- gético con el fin de comprender la sensibilidad de cada variable en la explicación de la $E T_{\text {real }}$ Se encontraron diferencias estadísticamente significativas con un nivel de significancia de 0,05 en los términos $R n, G$ y $u_{2}$, indicando la gran sensibilidad de los parámetros mencionados. La variable $R n$ es el término de mayor aporte en el balance de energía e, indirectamente, en el balance hídrico. Como se mencionó anteriormente, $G$ tiene mayor peso a escala horaria, quedando explicada la sensibilidad del parámetro. La diferencia entre ambos métodos en casos de valores bajos de $\mathrm{Hs}$ se explica por la sensibilidad de la diferencia $\left(T_{r a d}-T_{a}\right)$. En el análisis se observó que no tiene un peso significativo y considerable en el modelo energético, requiriendo un desarrollo más profundo de la Ecuación 7 y dejando en evidencia los errores en modelar este parámetro con una sola medida.

Por otra parte, en el modelo energético, el flujo de calor sensible es directamente proporcional a la velocidad del viento, que depende de factores como la topografía o la distribución de las cubiertas vegetales. Por este motivo, tener una red de medición espacialmente distribuida para este parámetro es complejo,

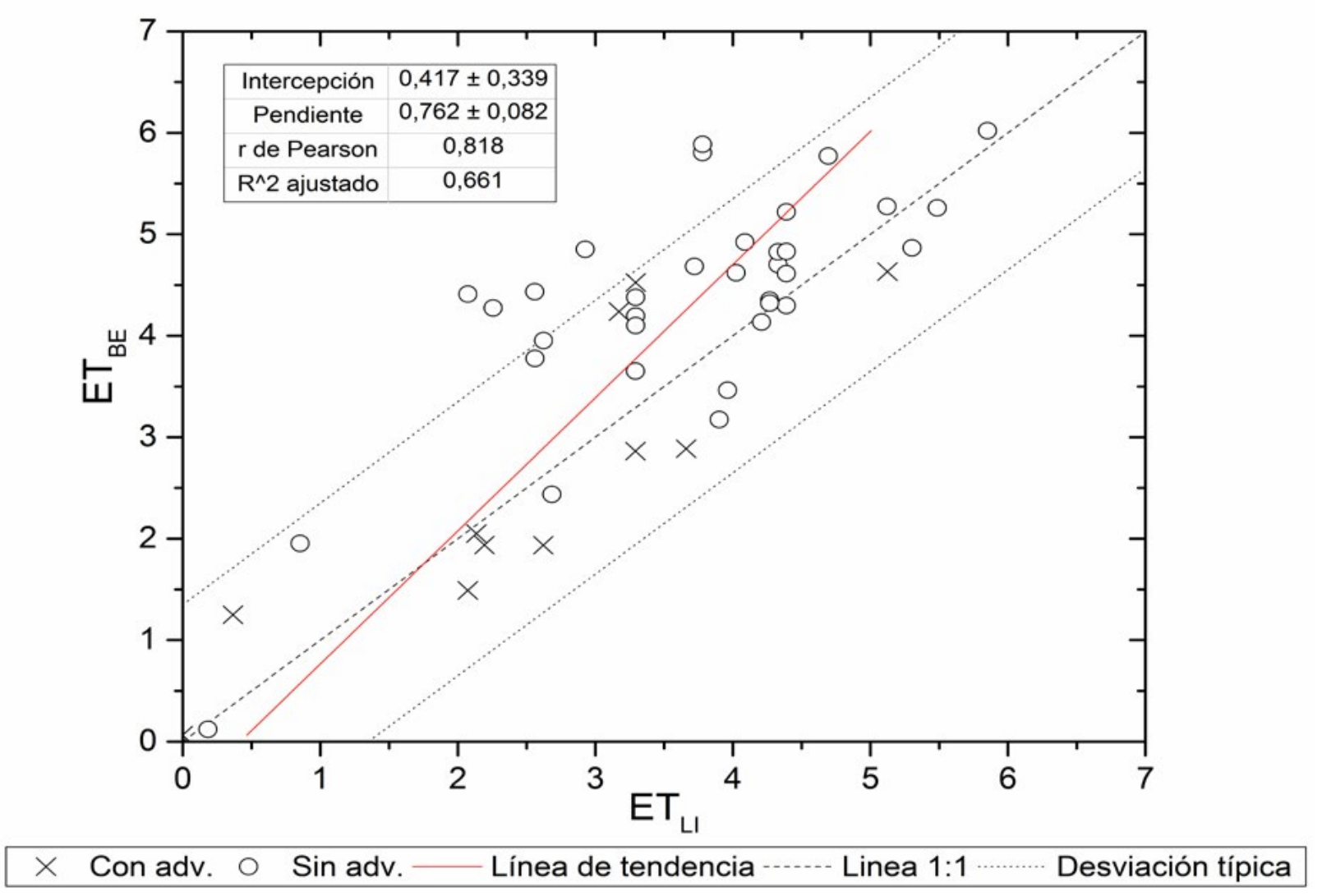

Figura 3. Comparación entre los valores diarios de ET obtenidos por el método de balance de energía y el lisímetro. Los datos se registraron en febrero, marzo y septiembre del 2017. Las líneas punteadas indican la recta 1:1 y la desviación estándar $(1,35 \mathrm{~mm}$ día-1).

Figure 3. Comparison between the daily ET values obtained by the energy balance method and the lysimeter. The data was recorded in February, March and September of 2017. The dotted lines is the 1: 1 line and standard deviation (1.35 mm day ${ }^{1)}$. 
pero se facilita en zonas de llanura. El método propuesto para el estudio, en estos ambientes, brinda información adecuada para la validación de modelos satelitales que utilizan resoluciones espaciales bajas. En otras palabras, se presenta una herramienta conveniente para la gestión y planificación del manejo hídrico que se le da a una cuenca de llanura.

\section{Conclusiones}

En este trabajo se comparó la evapotranspiración que se obtiene como término residual del método de balance de energía (método indirecto) y aquella medida in situ por medio de un lisímetro de pesada (método directo). El estudio se realizó con registros de una parcela con pastura de referencia (Allen et al. 1998), sin contribución del agua subterránea en el proceso de evapotranspiración y rodeado de un campo de avena que cumplía los mismos requerimientos. Para cumplir este requisito, se utilizaron datos de febrero, marzo y septiembre, abarcando dos campañas. Por medio de un seguimiento preciso de los datos registrados se observaron relaciones significativas entre los modelos, comprobando la aptitud del balance energético, principalmente la validez del modelo de un nivel. Se mostró que para medidas horarias el término $G$ no puede ser despreciado ya que se registraron valores cercanos al $18 \%$ de $R n$, parámetro de mayor aporte en el balance de energía. Se observaron diferencias de $0,10-0,30 \mathrm{~mm}$ hora $^{-1}$ entre los métodos para valores mínimos de humedad de suelo $\left(0,20 \mathrm{~m}^{3} \mathrm{~m}^{-3}\right.$ para suelos arcillosos). En condiciones de sequedad edáfica la energía disponible se pierde principalmente como flujo de calor sensible. Este término es directamente proporcional a la diferencia $\left(T_{r a d}-T_{a}\right)$, la cual no explicó la $E T_{\text {real }}$ por medio del modelo de una capa. Una corrección de la temperatura radiativa en estas condiciones mejoraría el modelo, sin embargo, se ofrece una alternativa simple para la validación de métodos indirectos a escala de región o cuenca. Para valores medios a máximos de humedad de suelo $\left(0,30-0,41 \mathrm{~m}^{3}\right.$ $\mathrm{m}^{-3}$ ) se observaron diferencias de 0,01-0,15 mm hora-1 entre los métodos, mostrando la efectividad para el cálculo de la $E T_{\text {real }}$ mediante el modelo de balance de energía simplificado. El método puede ser aplicado en ambientes similares, y en los casos en que el nivel freático se encuentre cercano a la superficie se deberá de mantener el nivel-capilaridad en el lisímetro para contemplar la influencia del agua subterránea en el proceso de evaporación y transpiración.

\section{Agradecimientos}

El trabajo se realizó en marco a una beca doctoral otorgada por la Consejo Nacional de Investigaciones Científicas y Técnicas de Argentina (CONICET), con fondos del proyecto FONARSEC 19 "Desarrollo e implementación de sistemas automáticos de alerta de inundaciones y sequías en el área sur de la cuenca del río Salado, provincia de Buenos Aires" (REDIMEC SRL - Autoridad del Agua - Comisión de Investigaciones Científicas de la Provincia de Buenos Aires), y fondos otorgados por la Agencia Nacional de Promoción Científica y Tecnológica de Argentina, PICT 2016-1486 - Proyecto EVAPAMPAS. Se agradece al MSc. Martín Bayala por la gran ayuda que ofreció para la construcción, instalación y puesta en marcha del lisímetro. Por último, agradecemos el aporte de los revisores anónimos y editores, los cuales ayudaron con sus observaciones en la mejora del manuscrito.

\section{Referencias}

Aboukhaled, A., Alfaro, A., \& Smith, M. 1982. Lysimeters. FAO Irrigation and Drainage paper $\mathrm{N}^{\circ} 39$. Roma.

Aliaga, V., Ferrelli, F., Piccolo, M. C. 2017. Regionalization of climate over the Argentine Pampas. International journal of climatology. Published online in Wiley Online Library. 37 (S1), 1237-1247.

Allen, R. G., Pereira, L. S., Raes, D., Smith, M. 1998. Crop evapotranspiration-Guidelines for computing crop water requirements. FAO Irrigation and drainage paper $\mathrm{N}^{\circ} 56$. Roma.

Allen, R. G., Pereira, L. S., Howell, T., Jensen, M. E. 2011, "Evapotranspiration information reporting: I. Factors governing measurement accuracy". Agricultural Water Management 98, 899-920

Allen, R. G., Pereira, L. S., Howell, T., Jensen, M. E. 2011. "Evapotranspiration information reporting: II. Recommended documentation". Agricultural Water Management 98, 921-929

Brutsaert, W. 2005. Hydrology, an Introduction. Cambridge University Press, Cambridge. http://dx.doi. org/10.1017/CBO9780511808470

Carmona, F., Rivas, R., Ocampo, D., Schirmbeck, J., Holzman, M. 2011. Sensores para la medición y validación de variables hidrológicas a escalas local y regional a partir del balance de energía. Aqua-LAC. 3 (1), $26-36$.

Carmona, F., Orte, P.F., Rivas, R., Wolfram, E., Kruse, E. 2018. Development and Analysis of a New Solar Radiation Atlas for Argentina from Ground-Based Measurements and CERES_SYN1deg data. Egyptian Journal of Remote Sensing and Space Science. Volume 21 (3), 211-217

Chow, V. T. 1994. Hidrología aplicada. McGraw-Hill ISBN: 958-600-171-7.

Dietrich, O., Fahle, M., Steidl, J. 2019. The Role of the Unsaturated Zone for Rainwater Retention and Runoff at a Drained Wetland Site. Water. 11 (7), 1404

Dormaar, J. F., Chanasyk, D. S. 1995. Effect of native prairie, crested wheatgrass (Agropyron cristatum (L.) Gaertn) and Russian wildrye (Elymus junceus Fisch.) on soil chemical properties. J. Range Manag., vol 48 (3), 258-263.

Fahle, M., Dietrich, O. 2014. Estimation of evapotranspiration using diurnal groundwater level fluctua- 
tions: Comparison of different approaches with groundwater lysimeter data, Water Resour. Res., 50, 273- 286, doi:10.1002/2013WR014472.

Faramiñán, A., Carmona, F., Rivas, R., Bayala, M. 2017. Medida directa de la evapotranspiración por medio de un lisímetro de pesada digital. XXVIII Reunión científica de la Asociación de Astrónomos, Geofísicos y Geodestas (AAGG), La plata, Argentina, 31.

Hannes, M., Wollschläger, U., Schrader, F., Durner, W., Gebler, S., Pütz, T., Fank, J., von Unold, G., and Vogel, H.-J. 2015. A comprehensive filtering scheme for high-resolution estimation of the water balance components from high-precision lysimeters, Hydrol. Earth Syst. Sci., 19, 3405-3418

Hoffmann, M., Schwartengräber, R., Wessolek, G., Peters, A. 2016. Comparison of simple rain gauge measurements with precision lysimeter data. Atmospheric Research 174 175, 120-123

Holzman, M., Rivas, R., Piccolo, M. C. 2014. Estimating soil moisture and the relationship with crop yield using surface temperature and vegetation index. International Journal of Applied Earth Observation and Geoinformation. 28, 181-192

Ibáñez Plana, M. 1998. Estimación de la evapotranspiración regional a partir de la Razón de Bowen radiativa. Tesis doctoral, Universitat de València, Valencia, España.

Ma, Z., Wang, W., Zhang, Z., Brunner, P., Wang, Z., Chen, L., Zhao, M., Gong, 2019. Assessing bare-soil evaporation from different water-table depths using lysimeters and a numerical model in the Ordos Basin, China. Hydrogeol Journal. https://doi. org/10.1007/s10040-019-02012-0

Ocampo, D. 2013. Estimación de la fracción evaporativa a partir de registros de humedad de suelo y un lisímetro de pesada. Encuentro del "International Center For Earth Sciences" - E-ICES 8E-ICES8.

Olías, M., Cruz San Julián, J.J., Benavente, J. Contribución de datos lisimétricos a la evaluación de la Recarga al Acuífero Almonte-Marismas. Geogaceta, 33, 107-110.

Parisi, S., Mariani, L., Cola, G., Maggiore, T. 2009. Mini-Lysimeters evapotranspiration measurments on suburban enviroment. Italian Journal of Agrometeorology, 3, 13-16.
Pereira, A. R. 2004. The Priestley-Taylor parameter and the decoupling factor for estimating reference evapotranspiration. Agricultural and Forest Meteorology $125,305-313$

Picard, A., Davis, R. S., Gläser, M., Fujii, K. 2008. Revised formula for the density of moist air (CIPM2007). Metrología, 45, 149

Rivas, R., Ocampo, D. 2009. Comportamiento del balance de energía en un cultivo de Avena sativa L. Estudios en la Zona no saturada del Suelo, Vol IX O. Silva et al. Barcelona.

Schirmbeck, Rivas, R. 2007. Comportamiento de los términos del balance de energía en una pastura. TELEDETECION, Hacia un mejor entendimiento de la dinámica global y regional. Martin ISBN: 978987-543-126-3.

Schrader, F., Durner, W., Fankb, J., Gebler, S., Pützc, T., Hannesd, M., Wollschläger, U. 2013. Estimating precipitation and actual evapotranspiration from precision lysimeter measurements. Four Decades of Progress in Monitoring and Modeling of Processes in the Soil-PlantAtmosphere System: Applications and Challenges. Procedia Environmental Sciences 19, 543-552

Schwaerzel, K., Bohl, H. P. 2003. An easily installable groundwater lysimeter to determine water balance components and hydraulic properties of peat soils. Hydrology and Earth System Sciences, 7(1), 23-32 Silicani, M. 2014. Diseño, construcción y operación de un lisímetro de bajo costo. Tesis de especialización, Universidad Nacional de Cuyo, Mendoza, Argentina.

Vásquez, V., Thomsen, A., Iversen, B. V., Jensen, R., Ringgaard, R., Schelde, K. 2015. Integrating lysimeter drainage and eddy covariance flux measurements in a groundwater recharge model, Hydrological Sciences Journal, 60 (9), 1520-1537

Weinzettel, P., Usunoff E. 2001. Cálculo de la recarga mediante la aplicación de la ecuación de Darcy en la zona no saturada. In Las caras del agua subterránea, Tomo II, Medina A, Carrera J (eds). Barcelona, España; 225-261.

Recibido: julio 2019

Revisado: noviembre 2019

Aceptado: enero 2020

Publicado: marzo 2021 